\title{
Yves Clot, Le travail à cœur : pour en finir avec les risques psychosociaux
}

Paris, La Découverte, coll. « Cahiers libres », 2010

\section{Serge Volkoff}

\section{OpenEdition}

\section{Journals}

\section{Édition électronique}

URL : https://journals.openedition.org/travailemploi/5678

DOI : $10.4000 /$ travailemploi.5678

ISSN : 1775-416X

\section{Éditeur}

DARES - Ministère du Travail

Édition imprimée

Date de publication : 15 juin 2012

Pagination : 93-94

ISSN : 0224-4365

\section{Référence électronique}

Serge Volkoff, "Yves Clot, Le travail à cœur : pour en finir avec les risques psychosociaux », Travail et Emploi [En ligne], 130 | avril-juin 2012, mis en ligne le 01 décembre 2012, consulté le 30 juillet 2021. URL : http://journals.openedition.org/travailemploi/5678; DOI : https://doi.org/10.4000/travailemploi. 5678

Ce document a été généré automatiquement le 30 juillet 2021.

(c) Direction de l'animation de la recherche, des études et des statistiques (Dares) 


\section{Yves Clot, Le travail à cœur : pour en finir avec les risques psychosociaux}

Paris, La Découverte, coll. « Cahiers libres », 2010

Serge Volkoff

\section{RÉFÉRENCE}

Yves Clot, Le travail à cœur : pour en finir avec les risques psychosociaux, Paris, La

Découverte, coll. «Cahiers libres », 2010, 190 p.

1 Sans déprécier la qualité scientifique de cet ouvrage, on peut saluer d'abord son intérêt comme recueil d'histoires. Sa capacité à convaincre, son accessibilité pour un public large, doivent beaucoup aux analyses de situations de travail que l'on trouve rassemblées. Celles-ci ont été menées par l'auteur lui-même, par des membres de son laboratoire, ou par d'autres chercheurs (en psychologie du travail, ergonomie, sociologie, etc.). Ces études, qui font l'objet d'une relecture attentive dans l'ouvrage, tiennent une place centrale: au sens propre, d'une part, puisque les deuxième et troisième parties - les plus développées de l'ouvrage qui en compte six - leur sont largement consacrées; en termes de démarche, d'autre part, car Yves Clot, comme souvent dans ses écrits, entend installer son propos dans « le temps continu du travail ordinaire » (p. 187).

2 Sont tour à tour conviés, au fil de ces chapitres, une ouvrière que rebute la qualité des farines utilisées désormais dans sa biscuiterie; des équipes de soignants hospitaliers dont la cohésion est mise à mal par les nouveaux indicateurs de performance; un professeur de mathématiques désarçonné par la perte de sens de sa mission éducative ; des guichetiers de La Poste à qui l'on enjoint de faire croître le chiffre d'affaires ; un agent commercial, narquois et amer d'avoir vendu une connexion internet à un client sans ordinateur; ou encore, des contrôleurs ferroviaires qui simulent des appels téléphoniques pour faire croire qu'un fraudeur récalcitrant va être sanctionné ; et bien d'autres. 
3 Les titres de ces deux chapitres, "La qualité empêchée » et «Le collectif en miettes », sont explicites, mais ne doivent pas prêter à simplification. L'objectif premier du livre n'est pas de dévoiler les « désordres du travail » (pour reprendre la formule de Philippe Askenazy, que l'auteur cite à plusieurs reprises). Yves Clot, comme d'autres, a contribué à dresser ce constat nécessaire mais son propos est ici surtout d'alerter sur les impasses et les dérives où pourraient se laisser porter les politiques publiques et les pratiques d'entreprises, quand celles-ci entendent remédier à ces désordres ou limiter au moins leurs méfaits.

4 La période s'y prête. Les thématiques du stress au travail, ou des risques psychosociaux, prennent de l'ampleur, alimentées par des épisodes tragiques : les suicides au travail dans plusieurs grandes entreprises ou administrations. Des dirigeants d'établissements, des parlementaires ou des membres du gouvernement ont jugé nécessaire de s'en saisir, et même d'agir vite. Or, écrit Yves Clot, «l'urgence est souvent la compensation factice d'une longue inaction, et il arrive souvent qu'elle complique l'action en préparant le retour à l'inertie»(p.9). La fébrilité est propice à l'émergence de stratégies palliatives dont l'ouvrage pointe, sur la base de rapports officiels ou de discours patronaux, les orientations que l'auteur juge discutables telles que la priorité accordée aux approches en termes d'affects et de relations interpersonnelles, la volonté de repérer les personnalités supposées fragiles ou le recours à des interventions « cicatrisantes».

5 Un véritable marché du « risque psychosocial sous-traité » (p. 29) s'est constitué sur ces bases. L'auteur énumère les actions-types auxquelles cette offensive donne lieu: instauration de numéros verts pour solliciter le soutien d'un psychologue clinicien; diffusion de critères de détection des salariés en difficulté ; activation des "signalants " pour favoriser cette détection; ouverture de droits à des consultations aux frais de l'employeur (les "tickets psy»). Yves Clot dispose de formules percutantes pour caractériser ces démarches, qui relèvent selon lui d'un "retraitement des déchets subjectifs du travail»(p.132), d'une «ouverture de couloir humanitaire dans l'organisation » (p. 135), voire d'un «néo-fordisme monté sur coussin compassionnel » (p. 145).

6 En s'exprimant ainsi, Yves Clot cherche moins à stigmatiser les responsables de ces pratiques qu'à désigner - en creux - les préoccupations majeures qui sont ainsi occultées: les questions du travail bien ou mal fait, les conflits légitimes que ces questions méritent d'alimenter, le potentiel créatif de discordances qu'il faudrait, justement, se garder d'estomper. Les récits commentés dont on a déjà parlé visent à démontrer que des démarches menées avec précaution et centrées sur une réhabilitation des disputes professionnelles sont à la fois nécessaires et faisables.

7 Au-delà des exemples, cette démonstration s'appuie sur un cadre théorique, que l'auteur a développé davantage dans ses écrits précédents, mais dont on retrouve ici les éléments clefs. Outre les recherches propres d'Yves Clot, ce cadre intègre les réflexions d'auteurs importants, de diverses disciplines, auxquels il aime à se référer: qu'il s'agisse de philosophes (Georges Canguilhem, Paul Ricœur, Guillaume Le Blanc), de psychologues ou de psychothérapeutes (Lev Vygotstki, François Tosquelles), d'ergonomes (Antoine Laville, François Daniellou), de l'économiste Marc Bartoli, du sociologue Philippe Zarifian, du médecin Philippe Davezies ou du préventeur Damien $\mathrm{Cru}$, Yves Clot convoque donc un faisceau d'apports théoriques qui appelle une lecture précise et qu'on ne résumera pas ici. Retenons que dans l'ouvrage ces apports sont 
mobilisés à l'appui d'une déconstruction de la notion même de « risque psychosocial », comme l'annonce le sous-titre du livre.

8 L'auteur oppose ainsi deux points de vue: «Une première approche générale du problème par "l'exposition" des salariés à un "risque" indéfini, et une seconde - tout aussi générale - qui s'attache à une autre exposition : celle de la qualité du travail à un conflit de critères dans le travail lui-même » (p. 105). Il opte pour la seconde, sans en masquer les difficultés. Cette approche renouvelée a des retombées concrètes: la prudence vis-à-vis des démarches hygiénistes, le souci de promouvoir une « entrée par la qualité du travail». Elle constitue aussi une contribution aux disputes professionnelles dans le métier même de psychologue, celui de l'auteur, et plus largement dans le réseau des chercheurs et experts dans ce domaine. On peut ici relever deux argumentaires.

D'une part, l'ouvrage questionne les évaluations quantitatives des facteurs de risque, et en particulier, les propositions émanant du Collège d'expertise, présidé par Michel Gollac, qui a remis ses conclusions en 2011 dans un rapport final donc postérieur à la rédaction de l'ouvrage. Yves Clot a été auditionné par ce Collège, mais n'a pas souhaité en être membre (à la différence de l'auteur de la présente note, juge et partie ici). Son appréciation des travaux du Collège, et des analyses chiffrées en général, est nuancée, et l'on en retiendra volontiers cette remarque importante: «J'ai pu mesurer à quel point la mise en débat de données chiffrées dans un collectif professionnel peut devenir une source de controverse et de pensée; mais dans ce cas le chiffre est pris comme objet d'une analyse en vue d'un diagnostic qu'il ne contient pas en puissance » (p. 127).

D'autre part, Yves Clot poursuit, dans le dernier chapitre du livre, sa réflexion critique vis-à-vis des thématiques de souffrance au travail et en particulier des orientations proposées par Christophe Dejours depuis une dizaine d'années, ou de celles qu'avance Emmanuel Renault dans ses recherches sur les «souffrances sociales». Ce passage, difficile à résumer, explicite les réserves d'Yves Clot notamment sur les interprétations en termes de « défaut de reconnaissance », ou d'une " anesthésie de la pensée » chez les salariés qui en sont victimes - ce qui, par réaction, assignerait avant tout aux « experts » une fonction de témoignage. L'auteur réaffirme sa volonté d'être attentif, au contraire, aux postures actives chez les travailleurs, à « leur désir de travailler malgré tout, qui tourne trop souvent mal, mais sans disparaître » (p. 156).

11 Le lecteur ne s'équipera pas ici en «bonnes pratiques", un terme qu'Yves Clot récuse avec raison. Les méthodes en clinique de l'activité sont en filigrane, mais leur présentation n'est pas l'objet du livre, et une initiation sommaire ne suffirait certainement pas pour se les approprier. En tout cas les chercheurs, praticiens, acteurs ou négociateurs dans le domaine de la santé au travail trouveront là de quoi progresser dans une réflexion sur leurs propres cadres d'action. 


\section{AUTEURS}

\section{SERGE VOLKOFF}

Centre d'études de l'emploi (CEE) - Centre de recherches et d'études sur l'âge et les populations au travail (CREAPT) 